\title{
중환자실에서의 결박
}

\author{
울산대학교 의과대학 서울아산병원 정신건강의학과 \\ 이 중 선
}

\section{Restraint in the Intensive Care Unit}

\author{
Jung Sun Lee, MD, PhD
}

Department of Psychiatry, Asan Medical Center, University of Ulsan College of Medicine, Seoul, Korea

An increasing number of patients are treated in the intensive care unit (ICU) due to advances in medicine. Usually, healthcare professionals decide on the patient's care in order to save the patient's life. Sometimes, these actions conflict with patients' rights and autonomy. For example, the application of restraint in incompetent patients in the ICU violates patients' rights to freedom and self-decision. We reviewed the definition, prevalence, patterns, predictors, and guideline of restraints in the ICU setting.

J Neurocrit Care 2015;8(2):73-77

Key Words: Restraint; Intensive care unit; Physical restraint; Guideline

\section{서 론}

의료 기술의 발달과 평균 수명 증가 등으로 중환자실에서 치료를 받는 환자들이 증가하고 있다. 중환자실에 입원한 환 자의 상태가 위중하기 때문에 중환자실 안에서 이루어지는 대 부분의 처치들을 의료진의 판단에 따라 '최선의 치료'에 도움 이 되는 방향으로 결정되고 시행된다. 이런 과정에서 일부 처 치들은 환자의 권리와 충돌하게 되며, 일부 환자와 보호자는 이런 처치의 당위성에 문제를 제기하기도 한다. 본 논문에서 는 그 동안 중환자실에서 자주 시행되고 있지만 많은 관심을 받지 못했던 결박(restraint) 개념과 현황을 살펴보고 이의 유용 성과 권장되는 절차에 대해 살펴 보도록 하겠다.

Received: October 28, 2015 / Revised: November 10, 2015

Accepted: November 10, 2015

Address for correspondence: Jung Sun Lee, MD, PhD

Department of Psychiatry, Asan Medical Center, University of Ulsan College of Medicine, 88 Olympic-ro 43-gil, Songpa-gu, Seoul 05505, Korea

Tel: +82-2-3010-3410, Fax: +82-2-485-8381

E-mail: js_lee@amc.seoul.kr

\section{본 론}

\section{결박의 개념}

결박은 환자의 움직이나 몸에 대한 접근을 제한하여 의학적 상태를 호전시키거나 부작용을 예방하는 치료행위로 정의할 수 있다. ${ }^{1}$ 결박에는 물리적 도구를 이용한 신체적 결박과 약물 (안정제, 마취제, 근육 이완제 등)을 이용한 화학적 결박이 있 다. 흔히 사용되는 물리적 결박 도구에는 조끼 결박대, 허리 결박대, 장갑 결박대, 손목 또는 발목 결박대, 팔꿈치 결박대, 전신 결박대 등이 있다.

\section{결박의 현황과 이유}

중환자실에서 환자에게 결박을 하게 되는 주된 이유는 시행 되는 치료 환경과 환자의 상태에 따라 다르지만 보통 자주 언 급되는 이유는 다음과 같다. 첫째, 환자가 의식적 또는 무의식 적으로 치료에 필요한 의료 장치를 제거하거나 치료 행위를 방해하는 경우가 있는데 이런 치료 방해 위험성을 줄이기 위 해서 결박을 하는 경우가 있다. 둘째, 의식이 명료하지 않은 상태에서 환자가 낙상을 당하거나, 스스로 의료 기구를 제거 하다가 발생할 수 있는 신체 손상을 예방하기 위한 것이다. 셋 째, 환자의 치료 방해와 신체적 손상으로 인한 중환자실 입원 
기간의 연장을 예방하기 위해 결박을 시행하기도 한다. ${ }^{1-4}$

실제로 미국의 49개 중환자실에서 시행된 대규모 전향적 연구에 의하면 중환자실 입원한 환자는 평균적으로 1000 patient-day 동안 22.1회 의료 장비를 스스로 제거를 하고, 이 중 $23 \%$ 에서 신체적 손상이 발생하였다고 한다. ${ }^{5} 24$ 시간 이 상 중환자실에 입원한 환자를 대상으로 한 연구에 의하면 $28 \%$ 환자가 의료 기구를 스스로 제거 했는데 그 중 $88 \%$ 는 GI tube 와 혈관 카테터였다. ${ }^{6}$ 미국, 아시아 및 유럽의 연구 결 과를 종합한 문헌고찰에 의하면 발관(extubation)의 incidence rate는 0.3-14.3\%이고 prevalence는 2-25.6/1000 ventilator-day 라고 한다. ${ }^{7}$

실제로 얼마나 많은 중환자실 환자가 결박을 경험하는지 조 사하는 것은 결박의 범위를 어디까지 볼 것인가에 따라 많이 다르다. 만약 포괄적인 화학적 결박의 하나로 볼 수 있는 진 정(sedation)을 포함하여 조사한다면 대략 $85 \%$ 의 환자가 중환 자실 입원 기간 중에 한 번 이상의 결박을 경험한다. ${ }^{8}$ 신체적 결박 비율은 치료 환경-국가, 의료진 비율, 내/외과계 중환자 실 여부 등-에 따라 많이 다른데 일반적으로 중환자실에서는 24-40\% 정도 되며 일반병동보다 훨씬 높다고 알려져 있다. ${ }^{3}$ 나라별로 구체적으로 살펴보면 독일의 경우 $12 \%$, 프랑스는 인공 호흡 환자의 $50 \%$ 이상에서 결박을 한 적이 있는 중환자 실은 전체 중환자실의 $82 \%$ 에 해당하였다. ${ }^{10}$ 유럽의 많은 병원 에서 시행된 전향적 연구인 PRICE(Physical Restraint in Intensive Care in Europe)에 의하면 33\% 환자가 중환자실 치료 기간 중에 결박된 적이 있었다. ${ }^{11}$ 우리나라의 종합병원 중환자실에 서의 결박대 적용 비율은 $31-46 \%{ }^{12-15}$ 로 조사되었고 신경외과 의 경우 빈도가 가장 높아 $48.4 \%$ 였다. 평균 결박 지속 시간은 113 시간 이었다. ${ }^{15}$ 우리나라 연구에 의하면 결박의 중요한 이 유는 기관내삽관과 같은 의료 보조 기구를 유지, 낙상 예방과 치료를 용이하게 하기 위해서 였다. ${ }^{12,16}$ 반면 결박 해제를 결정 하는 요인으로는 환자의 움직임 또는 인지 상태의 향상이라는 답변이 가장 많았다. ${ }^{12,17}$

주로 사용된 신체적 결박의 방법으로는 양손 결박이 59\%, 한쪽 손 결박이 $14 \%$, 조끼/자켓 결박이 $16 \%$ 그리고 한쪽 다 리 결박 $2 \%$, 양쪽 다리 결박이 $3 \%$ 였다. ${ }^{3}$ 우리나라의 경우 양 손 결박이 55-62\%, 한쪽 손 결박이 12-14\%, 한쪽 다리 결박 이 1\% 미만, 사지 결박이 19-26\%, 가슴과 사지결박은 4-6\%였 다. ${ }^{18}$ 우리나라 뿐만 아니라 외국 결과를 볼때 주로 상지에 대 한 결박이 많았다.

\section{결박의 위험인자}

여러 연구 결과를 종합해 보면 benzodiazepine, opioid, antipsychotics 하루 용량이 높을 수록, 초조 증상이 심할 수록 결
박 비율이 높았고 대학 소속 병원일 수록 비율이 낮았다..$^{19}$ 또 한 섬망, ${ }^{20}$ 니코틴 금단으로 인한 불안과 초조 ${ }^{21}$ 등이 있는 경 우 결박 비율이 높았다. PRICE 연구에서는 인공호홉기 사용, 진정되어 있거나, 의료 기관 규모가 크거나, 낮에 간호사/환자 비율이 낮은 곳에서 더 유의하게 결박 비율이 높은 것으로 조 사되었다. ${ }^{11}$

섬망은 중환자실에 입원 중인 환자 중 약 $32 \%$ 에서 발생하며 병원 전체 그리고 중환자실 입원기간을 늘리고 병원 및 중환 자실 사망률을 높인다고 한다..$^{22}$ 그리고 중환자실에서 결박을 시행하는 이유 중에 $14.7 \%$ 가 섬망 때문이라는 연구 결과를 고려할 때 중환자실에서 섬망 환자의 적절한 관리는 매우 중 요하다.

결박을 하기 2시간 전에 $74 \%$ 환자가 심한 초조 증상을 보였 다는 연구 결과 등을 고려할 때 중환자실에서 결박을 하게 되 는 주요한 원인 중에 하나는 초조(agitation)이다. 중환자실 입 원 환자 중에서 초조 증상을 보이는 비율은 52-70\% 정도였으 며 주로 입원 4.4 \pm 5.6일에 발생하였다. 그리고 평균 4일 정도 지속되었다고 한다. 그리고 초조 발생의 위험 요인으로는 입 원 당시 정신작용제(psychoactive drug)을 사용 중이거나, 알코 올 남용력이 있거나, 중환자실 입원 중에 진정제를 사용하는 경우, 패혈증, 기도 삽관, 수술 또는 진단적 처치, 불안, 수면 박탈, 인공 호흡기 세팅, 저산소증, 고탄산혈증(hypercapnia), 전해질 불균형 등이 있다. 또한 초조는 중환자실 입원 기간을 연장시키고 의도하지 않은 발관/혈관 카테터 제거의 위험성 을 높인다. ${ }^{23-25}$ 스스로 발관한 환자는 그렇지 않은 환자 보다 2 배 정도 초조가 많이 동반되어 있었고, 더 많은 안정제를 복용 하고 있었다. 특히 midazolam 사용한 환자에서 더 많이 발관 이 발생하였다. ${ }^{26}$ 이렇게 benzodiazepine 특히 midazolam을 사 용하는 환자에서 초조 및 발관이 많은 것은 paradoxical excitation과 관련이 있을 것으로 생각된다.

\section{결박의 부작용}

보통 알려져 있는 결박의 부작용으로는 생명기능(vital function)의 저하, 상완신경 손상 등 신경손상, 욕창, 흡인성 폐렴, 혈전색전증, 변비, 골절, 면역결박 등이 있다. 그리고 중환자 실의 입원 기간의 연장과 밀접한 관계가 있다. ${ }^{27-33}$

\section{결박에 대한 환자의 태도}

일반적으로 결박은 치료를 위해 시행되지만 환자에게 과도 한 스트레스를 주는 것이 아닌가 하는 걱정을 의료진들은 하 게 된다. 그래서 일부 연구에서는 간호사들은 신체적 결박보 다는 약물을 이용한 진정(sedation)을 선호한다고 조사되었 다. ${ }^{34}$ 실제로 결박되어 있는 환자 중에 의사소통이 가능한 환 
자 중 $33 \%$ 가 결박에 대해 부정적인 느낌을 호소하였고 ${ }^{30}$ 중환 자실에서 결박은 향후 외상후 스트레스 장애 발생과 관련이 있다는 연구도 있다. ${ }^{35,36}$

하지만 중환자실에 입원 중인 환자 중 35-60\% 환자에서 분 절된 기억과 기억력 저하가 발생하여 중환자실에서 있었던 일 을 잘 기억하지 못한다. ${ }^{37}-39$ 게다가 중환자실에 있었던 일을 기억하는 환자들 중에서 아무도 결박이 스트레스가 되었다고 진술하지는 않았다. ${ }^{39}$ 뿐만 아니라 문헌 고찰 연구에 의하면 연구 중에서 오직 2개의 연구에서만 환자들이 손이 결박되어 있어 좌절감과 불안, 불편감을 느꼈다고 기술하였다. ${ }^{40}$ 결박된 환자가 저하된 기억력과 주의 집중력으로 상황을 잘 기억 못 할 수도 있지만 일부에서는 특히 의사소통이 가능한 환자의 경우 결박된 상황을 스트레스로 받아들일 가능성이 있다. 따 라서 후반부에 언급되겠지만, 결박된 환자에게 지속적으로 결 박의 이유에 대해서 설명해 주는 것이 필요하다.

\section{결박의 효용성}

지금까지 살펴본 바에 따르면 다양한 이유로 중환자실에서 결박이 광범위하게 시행되고 있으나 다행히 환자들은 기억을 잘 못하거나 그 필요성을 인정하고 불편감을 크게 호소하지는 않았다. 그러나 결박이 과연 원래의 목적대로 환자를 보호하 고 치료에 도움이 되는지 살펴볼 필요가 있다.

결박을 하는 것이 환자의 발관이나 각종 라인의 제거를 예 방하는데 도움이 된다는 연구 결과도 있지만, ${ }^{41}$ 임의로 발관했 던 환자의 $1 / 3$ 에서 결박된 상태에서 발생했고, ${ }^{7}$ 오히려 결박 자체가 발관의 위험 요인이 된다는 연구 결과도 있다. ${ }^{42,43}$ 또 환자의 초조 증상을 치료하기 위해 약물로 진정시키는 경우가 있는데 이 역시 방법과 정도에 따라 섬망과 초조 증상이 더 심 해질 수 있다. ${ }^{44}$ 이와 같이 결박이 상황에 따라 목적을 달성하 지 못하고 환자에게 불편을 초래하고 부작용만 발생시킬 가능 성도 있다. ${ }^{4,45}$

\section{결박의 대안, 바람직한 결박 방법 및 절차}

결박을 하지 않고 환자를 안전하게 치료 할 수 있다면 가장 이상적이라고 할 수 있다. 지금까지 신체적 결박을 대신할 수 있는 여러 방법들이 알려져 있고 여기서 그 일부를 소개 하겠 다. 다만 연구가 부족하여 그 처치의 유용성에 대해서 결론을 내리기 어렵다.

환자를 불편하게 하거나 초조를 유발할 수 있는 요인 - 요 정체, 잘못된 위치의 기도 삽관, 불편한 자세- 등을 교정하는 것이 도움이 될 수 있다. 환자의 생각을 돌릴 수 있도록 음악 을 들려 주는 것도 도움이 된다. 중환자실 소음을 줄여 주고, 규칙적인 활동을 제공하고, 낮익은 가족의 간병 시간을 늘리
고, 자주 지남력 정보 제공하는 것이 좋다. ${ }^{1}$ 또한 환자의 초조 나 불안, 통증 등을 경감 시켜 줄 수 있는 약은 결박 감소에 효 과가 있다고 한다..$^{46}$ 또한 가급적 benzodiazepine 사용을 줄이 고 dexmedetomidine을 사용하면 기계환기기 사용 시간과 중환 자실 입원 기간을 줄일 수 있다고 한다. ${ }^{44}$

또한 가급적 각종 의료 기구의 필요성이 있는지 검토를 해 서 조기에 기구를 제거 하는 것이 좋다. 그리고 기구를 잘 고 정을 하고 환자가 쉽게 알아 볼 수 없게 숨기는 것도 좋은 방 법이다.

지금까지 살펴본 결박을 대신할 수 있는 방법으로 환자의 행동을 조절할 수 없다면 결박을 고려해야 하는데 그 과정에 서 몇가지 고려되어야 할 부분이 있다. 우리나라뿐만 아니라 많은 나라에서 결박의 결정이 주로 간호사에 의해 이루어지고 있으며 ${ }^{16}$ 결박의 시행과 방법 및 절차 그리고 필요성에 대한 의무기록 작성이 부족하고 일부 연구에서는 기록이 전혀 없는 경우도 있다고 한다. 프랑스의 130 개 중환자실 중에서 $21 \%$ 만 결박 처치에 대한 내부 규정을 가지고 있었다. ${ }^{10}$ 게다가 우리 나라의 경우 이런 의료 행위에 대해서 법률 조항이 모호하여 자칫 분쟁의 소지가 될 수 있다. 정신건강의학과 환자의 경우 정신보건법에 명확하게 결박의 목적과 절차가 기술되어 있어 법률적 근거가 되고 있다. 따라서 가급적 결박 이전에 결박 가 능성과 이로 인한 부작용 발생 가능성에 대해 설명하고 환자 나 보호자에게 동의서를 받는 것이 도움이 될 수 있다.

앞에서 살펴본 것처럼 결박의 빈도나 효용성이 환자의 상 태 및 진료 환경 등의 영향을 많이 받기 때문에 일률적인 결박 지침을 정하는 것이 사실 불가능할 수도 있다. 하지만 최상의 진료를 위해 참고 목적으로 American College of Critical Care Medicine, the Society of Critical Care Medicine, and the American Association of Critical Care Nurses에서 개발된 지침과 우 리나라 보건복지부가 정신건강의학과 환자를 위해 제시한 정 신보건 사업 안내 2012판에서 제시하는 중요한 지침을 소개하 겠다.

- 1 시간 마다 vital sign(혈압, 맥박, 호흡)을 확인하고 환자의 혈액 순환을 확인하기 위해 피부의 색깔이나 맥박을 확인 해야 한다. 그리고 사지의 움직이나 감각을 확인해야 한다.

- 최소 2 시간 간격으로 환자의 생리적 욕구를 확인하고 제공 해야 하며, 사지의 passive range of motion (ROM) 운동을 해 야 한다.

- 결박의 필요성이 기록되어야 한다.

- 결박 지시는 24시간을 넘기지 않아야 한다. 최소 8시간마다 결박을 중단하거나 단계를 낮출 수 있는지 검토해야 한다.

- 환자는 최소 4 시간마다 부작용 발생 여부를 확인해야 하 며, 특히 초조 증상이 있거나 다른 임상적 이유가 있다면 
더 자주 관찰해야 한다. 이런 관찰 내용은 기록으로 보존 해야 한다.

- 환자와 주요한 보호자는 지속적으로 결박의 필요성과 결박 에 대해 교육받아야 한다.

- 환자의 통증, 불안, 정신병적 증상 치료를 위한 마취제, 안 정제, 신경이완제는 결박 치료의 필요성을 감소 시키기 위 해 사용되어야 하며 화학적 결박을 위해서 과량 사용하면 안된다.

- 신경근차단제(neuromuscular blocker)는 화학적 결박 목적으 로 사용해서는 안되며, 장기적인 마비를 예방하기 위해 더 자주 관찰되어야 한다.

\section{결 론}

결박의 문제는 환자나 보호자가 의료진에게 허용한 환자를 위한 최선의 행동과 환자의 법적 또는 개인적 권리와 충돌을 할 수 있다. 따라서 미리 결박의 가능성에 대해 환자나 보호자 에게 설명을 하고 결박을 줄이기 위한 다양한 방법을 먼저 시 도해 보아야 하며 결박을 하더라도 최소의 범위에서 가급적 정해진 규정에 따라 시행되는 것이 좋을 것으로 생각된다.

\section{REFERENCES}

1. Maccioli GA, Dorman T, Brown BR, Mazuski JE, McLean BA, Kuszaj JM, et al. Clinical practice guidelines for the maintenance of patient physical safety in the intensive care unit: use of restraining therapies-American College of Critical Care Medicine Task Force 2001-2002. Crit Care Med 2003;31(11):2665.

2. Birkett KM, Southerland KA, Leslie GD. Reporting unplanned extubation. Intensive Crit Care Nurs 2005;21:65-75.

3. Minnick AF, Mion LC, Leipzig R, Lamb K, Palmer RM. Prevalence and patterns of physical restraint use in the acute care setting. J Nurs Adm 1998;28:19-24.

4. Bray K, Hill K, Robson W, Leaver G, Walker N, O'Leary $\mathrm{M}$, et al. British Association of Critical Care Nurses position statement on the use of restraint in adult critical care units. Nurs Crit Care 2004;9:199-212.

5. Mion LC, Minnick AF, Leipzig RM, Catrambone CD, Johnson ME. Patient-initiated device removal in intensive care units: a national prevalence study. Critical Care Medicine 2007;35:2714-20; quiz 2725.

6. Fraser GL, Riker RR, Prato S, Wilkins ML. The frequency and cost of patient-initiated device removal in the ICU. Pharmacotherapy 2001;21:1-6.

7. Bassi E, Ceresola M. Use of physical restraints in adult ICU patients to prevent patient-initiated device removal: a systematic review. The JBI Database of Systematic Reviews and
Implementation Reports 2011;9(32 Suppl):S237-50.

8. Woods JC, Mion LC, Connor JT, Viray F, Jahan L, Huber C, et al. Severe agitation among ventilated medical intensive care unit patients: frequency, characteristics and outcomes. Intensive Care Med 2004;30:1066-72.

9. Krüger C, Mayer H, Haastert B, Meyer G. Use of physical restraints in acute hospitals in Germany: a multi-centre crosssectional study. Int J Nurs Stud 2013;50:1599-606.

10. De Jonghe B, Constantin JM, Chanques G, Capdevila X, Lefrant JY, Outin H, et al. Physical restraint in mechanically ventilated ICU patients: a survey of French practice. Intensive Care Med 2012;39:31-7.

11. Benbenbishty J, Adam S, Endacott R. Physical restraint use in intensive care units across Europe: The PRICE study. Intensive Crit Care Nurs 2010;26:241-5.

12. Choi E, Song M. Physical restraint use in a Korean ICU. $J$ Clin Nurs 2003;12:651-9.

13. Cho Y, Kim J, Kim N, Choi H, Cho J, Lee H, et al. A Study on the use of physical restraints in ICUs. J Korean Acad Adult Nurs 2006;18:543-52.

14. Yeo JM, Park MH. Effects of on education program for nurses on the use of restraints. Taehan Kanho Hakhoe Chi 2006;36:532-41.

15. Kim MY, Park JS. A study on the application of physical restraints in intensive care units. J Korean Acad Fundam Nurs 2010;17:177-86.

16. Lee EG, Cho EH, Kim SA, Kim JK, Kim HM, Kim HE, et al. The study on physical restraint use in intensive care unit (ICU). Korean Clin Nurs Res 2003;8:117-30.

17. Titsworth WL, Hester J, Correia T, Reed R, Guin P, Archibald $\mathrm{L}$, et al. The effect of increased mobility on morbidity in the neurointensive care unit. J Neurosurg 2012;116:1379-88.

18. Kim YH, Jeong YS, Park JH, Yoon SH. The effects of nurse education on physical restraint use in the ICU. Korean J Anesthesiol 2008;55:590-5.

19. Luk E, Sneyers B, Rose L, Perreault MM, Williamson DR, Mehta S, et al. Predictors of physical restraint use in Canadian intensive care units. Crit Care 2014;18:R46.

20. Micek ST, Anand NJ, Laible BR, Shannon WD, Kollef MH. Delirium as detected by the CAM-ICU predicts restraint use among mechanically ventilated medical patients. Crit Care Med 2005;33:1260-65.

21. Lucidarme O, Seguin A, Daubin C, Ramakers M, Terzi N, Beck P, et al. Nicotine withdrawal and agitation in ventilated critically ill patients. Crit Care 2010;14:R58.

22. Ouimet S, Kavanagh BP, Gottfried SB, Skrobik Y. Incidence, risk factors and consequences of ICU delirium. Intensive Care Med 2006;33:66-73.

23. Jaber S, Chanques G, Altairac C, Sebbane M, Vergne C, Perrigault $\mathrm{PF}$, et al. A Prospective study of agitation in a medicalsurgical ICU: incidence, risk factors, and outcomes. Chest 2005;128:2749-57.

24. Fraser GL, Prato BS, Riker RR, Berthiaume D, Wilkins ML. Frequency, severity, and treatment of agitation in young ver- 
sus elderly patients in the ICU. Pharmacotherapy 2000;20:7582.

25. Treggiari-Venzi M, Borgeat A, Fuchs-Buder T, Gachoud JP, Suter PM. Overnight sedation with midazolam or propofol in the ICU: effects on sleep quality, anxiety and depression. Intensive Care Med 1996;22:1186-90.

26. Tung A, Tadimeti L, Caruana-Montaldo B, Atkins PM, Mion LC, Palmer RM, et al. The relationship of sedation to deliberate self-extubation. J Clin Anesth 2001;13:24-9.

27. Burchardi H, Schneider H. Economic aspects of severe sepsis: a review of intensive care unit costs, cost of illness and cost effectiveness of therapy. Pharmacoeconomics 2004;22:793813.

28. Prielipp RC, Coursin DB, Wood KE, Murray MJ. Complications associated with sedative and neuromuscular blocking drugs in critically ill patients. Crit Care Clin 1995;11:9831003.

29. Hofs $\varnothing$ K, Coyer FM. Part 2. Chemical and physical restraints in the management of mechanically ventilated patients in the ICU: a patient perspective. Intensive Crit Care Nurs 2007;23:316-22.

30. Mion LC, Frengley JD, Jakovcic CA, Marino JA. A further exploration of the use of physical restraints in hospitalized patients. J Am Geriatr Soc 1989;37:949-56.

31. Park YJ, Kim SJ, Park GC. A case of brachial plexus injury dueto physical restraint. J Korean Soc Emerg Med 2006;17:500-4.

32. Dickson BC, Pollanen MS. Fatal thromboembolic disease: a risk in physically restrained psychiatric patients. J Forensic Leg Med 2009;16:284-6.

33. Shehabi Y, Ruettimann U, Adamson H, Innes R, Ickeringill M. Dexmedetomidine infusion for more than 24 hours in critically ill patients: sedative and cardiovascular effects. Intensive Care Med 2004;30:2188-96.

34. Happ MB. Preventing treatment interference: the nurse's role in maintaining technologic devices. Heart Lung 2000;29:609.
35. Jones C, Bäckman C, Capuzzo M, Flaatten H, Rylander C, Griffiths RD. Precipitants of post-traumatic stress disorder following intensive care: a hypothesis generating study of diversity in care. Intensive Care Med 2007;33:978-85.

36. Micek ST, Anand NJ, Laible BR, Shannon WD, Kollef MH. Delirium as detected by the CAM-ICU predicts restraint use among mechanically ventilated medical patients. Crit Care Med 2005;33:1260-5.

37. Samuelson K, Lundberg D, Fridlund B. Memory in relation to depth of sedation in adult mechanically ventilated intensive care patients. Intensive Care Med 2006;32:660-7.

38. Capuzzo M, Pinamonti A, Cingolani E, Grassi L, Bianconi M, Contu $\mathrm{P}$, et al. Analgesia, sedation, and memory of intensive care. J Crit Care 2001;16:83-9.

39. Minnick A, Leipzig RM, Johnson ME. Elderly patients' reports of physical restraint experiences in intensive care units. Am J Crit Care 2001;10:168-71.

40. Stein-Parbury J, McKinley S. Patients' experiences of being in an intensive care unit: a select literature review. Am J Crit Care 2000;9:20-7.

41. Carrión MI, Ayuso D, Marcos M, Paz Robles M, de la Cal MA, Alía I, et al. Accidental removal of endotracheal and nasogastric tubes and intravascular catheters. Crit Care Med 2000;28:63-6.

42. Chang LY, Wang KW, Chao YF. Influence of physical restraint on unplanned extubation of adult intensive care patients: a case-control study. Am J Crit Care 2008;17:408-15; quiz 416.

43. Curry K, Cobb S, Kutash M, Diggs C. Characteristics associated with unplanned extubations in a surgical intensive care unit. Am J Crit Care 2008;17:45-51; quiz 52.

44. Wunsch H, Kress JP. A new era for sedation in ICU patients. JAMA 2009;301:542-544.

45. Ofoegbu BN, Playfor SD. The use of physical restraints on paediatric intensive care units. Paediatr Anaesth 2005;15:407-11.

46. Sessler CN, Varney K. Patient-focused sedation and analgesia in the ICU. Chest 2008;133:552-65. 